\title{
A 'rough guide' to publication
}

\author{
Submitting or resubmitting a manuscript-some important steps and decisions along the way.
}

The path to publication is a well-beaten one for some scientists but seems more like a dark, unmarked road to others. It helps to know what to expect from peer review (see our May 2006 editorial), but a number of other procedural steps often cause disorientation as well. Here are some trail blazes and travel advice.

Presubmission inquiry. Such an inquiry-typically a referenced abstract and brief description of the paper-is an optional step in Nature Methods' submission process, but is by no means necessary. It is an informal consultation and not a commitment on the part of either authors or editors.

Responding to reviewers' comments. Our advice is simple: tackle the scientific substance, and do it comprehensively. The best way to address reviewers' concerns is almost always to add data-this is far more effective than dismissing the concern, stating that it is beyond the scope of the paper, or making a cosmetic change.

We strongly recommend that you make a comprehensive attempt at addressing all the main points made by reviewers and editors, rather than trying to guess what the minimum required change will be. Reviewers' time is a limited resource that editors must conserve. We may refuse to bother the reviewers with a new version if we believe that their original comments have not been genuinely addressed.

To speed up evaluation of the revision, modifications should be underlined in the manuscript and accompanied by a point-by-point response to the referees' comments. The best responses consist of a copy of the reviewers' report in which the authors insert an answer to each point, explaining what was done to address the criticism and where in the manuscript the changes can be found.

Obviously, it does not hurt to be polite. If a reviewer's comment clearly indicates a misunderstanding, it is adequate to explain this, but an aggressive tone will not help. Rather, any misunderstanding in review should signal that the manuscript may not be as clear as you had thought and prompt you to clarify it to avoid similar confusion for future readers.
Dealing with rejection. We receive many more submissions than we can publish, and $80-90 \%$ of manuscripts are rejected either before or after external peer review. In some cases, although we cannot offer publication or review of a manuscript because it falls short of the journal's standards, we will indicate that we would be happy to look at a revised manuscript provided additional experimental data address specific limitations. We do mean it: we will reconsider a revised manuscript without prejudice. But there is no guarantee that it will be published or even sent to review. It depends on how convincing the new data are and what has been published in the interim. If the additional experiments take a long time to complete, the novelty of the information may wear off. Thus authors must carefully evaluate the requirements and weigh the risk, factoring in resources and existing data.

Appeals. If you are not invited to submit a revised manuscript and disagree fundamentally with our decision or the reviewers' criticisms, you may appeal the decision. But it is wise to consider this option carefully, for several reasons. First, only a minority of appeals lead to a reversal of the decision and, ultimately, to publication. Second, the appeal process can be long. We examine appeals thoroughly, but they must take second place to the normal workload. If the manuscript is reconsidered, it is likely to go through additional rounds of revision and review, sometimes with new referees who may bring up a new set of problems.

This being said, we do our best to be fair, and we are willing to reconsider a decision if you can convince us that there was a serious mistake: because you have additional data that address the major criticisms, because you can point to factual errors that affected the decision, or because you have persuasive evidence of referee bias. But merely reaffirming the importance of the work or arguing minor points is unlikely to be effective.

At each of these crossroads, a decision on our part will lead to one on yours about what to do next and how. We hope these insider views help you take the high road. 